\title{
INTERACTION OF SOUND FROM SUPERSONIC JETS WITH NEARBY STRUCTURES
}

\author{
C. C. Fenno, Jr. ${ }^{1}$ \\ National Research Council, Hampton, VA 23681-0001 \\ A. Bayliss ${ }^{2}$ \\ Northwestern University, Evanston, IL 60208 \\ L. Maestrello \\ NASA Langley Research Center, Hampton, VA 23681-0001
}

\begin{abstract}
A model of sound generated in an ideally expanded supersonic (Mach 2) jet is solved numerically. Two configurations are considered; (i) a free jet and (ii) an installed jet with a nearby array of flexible aircraft type panels. In the later case the panels vibrate in response to loading by sound from the jet and the full coupling between the panels and the jet is considered, accounting for panel response and radiation. The long time behavior of the jet is considered. Results for near field and far field disturbance, the far field pressure and the vibration of and radiation from the panels are presented. Panel response crucially depends on the location of the panels. Panels located upstream of the Mach cone are subject to a low level, nearly continuous spectral excitation and consequently exhibit a low level, relatively continuous spectral response. In contrast, panels located within the Mach cone are subject to a significant loading due to the intense Mach wave radiation of sound and exhibit a large, relatively peaked spectral response centered around the peak frequency of sound radiation. The panels radiate in a similar fashion to the sound in the jet, in particular exhibiting a relatively peaked spectral response at approximately the Mach angle from the bounding wall.
\end{abstract}

\footnotetext{
${ }^{1}$ Supported by NASA Langley Research Center while in residence under a National Research Council Postdoctoral Research Associateship Award.

${ }^{2}$ Partially supported by NASA Langley Research Center under contract NAS1-19480 while in residence at ICASE. Additional support was provided by NSF grants DMS 93-01635 and DMS 95-30937.
} 


\section{Introduction}

In this paper, the generation and propagation of sound in a supersonic jet are simulated (i) when the jet is free standing and (ii) when the jet is installed near an array of flexible aircraft type panels. In both cases a two dimensional jet is considered exiting from a converging-diverging (CD) nozzle extending to infinity in the upstream direction. In the installation case, a model is employed in which the unsteady flow field in the jet is fully coupled to the panel response and radiation. The jet is assumed to be ideally expanded in the steady state.

The primary objectives are (i) to characterize sound generation mechanisms and propagation phenomena in an ideally expanded supersonic jet and (ii) to characterize panel response and radiation under excitation by sound from the jet. The jet is initially excited by a spatially and temporally localized source of transient mass injection. This leads to an initial acoustic disturbance which propagates through the jet. As a result of the excitation, instability waves are generated in the jet. These waves grow and then decay as they convect downstream, generating sound in the process. This phenomenon occurs over time scales much longer than that of the excitation pulse. The long time response of the jet is considered here and data is only presented after the wave due to the excitation pulse has exited the domain of interest. Thus, instability wave generated sound in the jet is simulated. The simulation does not directly account for sound generated by small scale turbulent sources in the jet.

In previous work, jet acoustics, panel response and radiation have been considered for subsonic jets.[1, 2, 3] It was shown that for low subsonic jets the acoustic response of the jet exhibited a nearly continuous spectrum and the panels acted as filters converting the broadband forcing into relatively narrow spectral bands. The effect of forward motion on low speed jets was shown to reduce the level of the convective instability waves and thereby reduce the panel response and radiation. It was shown that in contrast to the behavior for low subsonic jets, high subsonic jets exhibited a relatively peaked spectrum with a peak frequency, $f_{*}$, occurring along with peak far field radiation at about $30^{\circ}$ from the jet axis. This behavior of the jet forcing was reflected in a similar panel response.

The exact sources of jet noise have been identified from the basic equations of fluid dynamics.[4, 5, 6, 7] Generally, these exact sources must be modeled in some way for the computation, e.g., to compute the loading on nearby panels. One way to enhance modeling of the sources is to separate out the different effects that lead to sound generation in a jet. In this paper sound generated from large scale instability waves is considered. Small scale turbulent structures are ignored. For supersonic jets it is known that the highest level of sound generated by instability waves convecting along the jet tends to propagate primarily at the Mach angle[8,9] and is thus referred to as Mach wave radiation. Away from the Mach angle the radiated pressure is at a low level and exhibits a nearly continuous spectrum. Supersonic jets operated under non-ideal conditions also exhibit shock cell induced noise, an effect not considered here. In this paper only shock-free jets (at least in the steady state) are considered.

Instability waves or large scale structures act as sources of sound in a jet. This has been shown in experiments[10, 11, 12] and studied by analytical[13, 14, 15, 16] and numerical[12, 17, 18] methods. A modified version of the Euler equations is employed to calculate the long time response of the excited jet. As a result, the inviscid sources of jet sound are 
computed directly together with the resulting sound generation. The sound radiation from the jet, together with the panel response and radiation when the sound excites nearby flexible panels are computed.

The computational domain is shown in Figure 1. A modified version of the Euler equations is solved in two domains; the jet domain and the radiation domain. These domains are separated by an array of six flexible panels. Panel response and radiation are also computed and are fully coupled to the fluid dynamics in the sense that at each timestep the fluid dynamics (Euler) computation provides the pressure difference across the panels, thereby allowing computation of panel displacement and velocity. The resulting panel velocity then serves as a boundary condition for the Euler computation. Thus, the panel excitation is obtained in a self-consistent manner directly from the Euler code, rather than from additional models. Similar computations for boundary layers and for panels excited by large amplitude acoustic disturbances in an ambient medium have also been performed [19, 20].

\section{Model and Numerical Method}

Referring to Figure 1, unsteady pressure, density and velocity are computed in both the jet and radiation domains. The jet domain simulates the aircraft exterior while the radiation domain simulates the aircraft interior. In the jet domain, the jet, exiting from a CD nozzle of width $D$, is excited by a spatially and temporally localized source of mass injection. This leads to the generation of a train of instability waves which propagate along the jet, decaying beyond the potential core of the jet and generating sound. The sound serves to excite the panels, leading to panel vibration and the radiation of sound into both the jet and radiation domain (i.e., in a real aircraft into both the exterior and interior). It is difficult to identify panel radiation in the jet domain, as the panel radiation is small compared to the sound generated within the jet. Therefore panel radiation in the radiation domain is studied where the panels are the only sources of sound.

The wall boundary between the two domains consists of six flexible panels as indicated in Figure 1. The panels are rigidly clamped to stringers separating any two adjacent panels and the wall is assumed to extend rigidly to infinity in both directions beyond the panels. The panels will be referred to as panels 1-6, numbered in ascending order as the downstream distance increases.

The computation of the nonlinear beam equation governing the panel responses is fully coupled to an Euler computation performed in both the jet and radiation domains. At each time step the pressure difference across the panels, obtained from the Euler computations, serves as a forcing term for the beam equation. The displacement obtained from the beam equation is differentiated in time and is then employed as a boundary condition on the normal velocity for the Euler computation. In the Euler computations the deflection of each panel is assumed to be small relative to the Euler length scales so the Euler boundary is treated as a horizontal line. The Euler computations employ a (2-4) version of the MacCormack scheme.[22] Second order finite differences combined with semi-implicit time differencing are used to solve the beam equation for each panel. Further details on both the coupling and the numerical scheme are given in the references. $[1,2,3]$ 
The Euler equations are solved in conservation form for the vector

$$
\hat{\mathbf{w}}=(\rho, \rho u, \rho v, E)^{T},
$$

where $\rho$ is the density, $u, v$ are the $x$ and $y$ components of the velocity respectively and $E$ is the total energy per unit volume,

$$
E=\frac{1}{2} \rho\left(u^{2}+v^{2}\right)+c_{v} \rho \tilde{T}
$$

where $\tilde{T}$ is the temperature and $c_{v}$ is the specific heat per unit volume. The pressure, $p$, is obtained from the equation of state.

The Euler equations are modified in the jet domain to account for the jet flow. The jet exits from a nozzle of width $D$ and the solution is computed both within and exterior to the nozzle. The Euler equations are modified to account for two different non-homogeneous forcing terms. [3] One term serves as an excitation pulse to excite the jet. It corresponds to a localized source of mass injection at the location $\left(x_{s}, y_{j}\right)$, where $y_{j}$ is the location of the jet axis and $x_{s}$ is approximately $1 D$ downstream from the nozzle exit. An alternative approach, involving time harmonic excitation of the jet is described by Mankbadi et al.[21] The second forcing term is designed so that in the absence of the starter pulse the solution to the Euler equations would be a stationary profile corresponding to a spreading jet. Mean profiles for $U, V, \rho$ and $T$ are employed and are described in more detail elsewhere.[3] The inclusion of this term separates the computation of the disturbance, in particular the resulting instability waves, from the computation of the mean flow (i.e. the spreading jet). Thus, the resulting system of equations allows for the simulation of instability waves and the resulting sound generation, together with the propagation of acoustic waves in the jet flow field, without requiring the computation of the spreading jet itself. Although this is a simplified model, it captures many of the observed features of instability wave generated jet sound and permits high resolution computation of the coupling of jet noise with the flexible panels and the resulting radiation from the panels. In particular, the model allows for computation of the natural sources of jet noise (the instability waves) together with the sound radiated by these sources.

Radiation boundary conditions are employed on all exterior boundaries except for the nozzle inflow where characteristic boundary conditions are employed. These are described in detail elsewhere.[3]

\section{Results}

The computations include both the near field (including jet flow instabilities) and far field acoustic jet response, unsteady disturbances in the nozzle, the responses of each of the panels to excitation from jet disturbances and radiation from the vibrating panels. The long time behavior of disturbances is considered, in order to distinguish intrinsic properties of the jet from the frequency spectrum of the excitation pulse (the results do depend on the amplitude of the excitation pulse). Results are presented for two computations, one for a free jet and one for an installed jet located approximately $7 D$ from the wall. In each case the computational domain extends $90 D$ downstream from the nozzle exit and $60 D$ upstream. 
In the free jet computation the domain extends $30 D$ from the jet in both the positive and negative $y$ directions. For the installed jet computation, the vertical domain extends $60 \mathrm{D}$ from the wall. There are no discernible boundary reflections over the timescales for which the data is presented. To further eliminate the effect of the boundaries, data is only considered for a region bounded away from the boundaries. The results have been validated by grid refinements. All results presented are for a jet with an exit Mach number of 2.0.

\section{$3.1 \quad$ Free Jet}

Figure 2 shows contours of unsteady pressure $\tilde{p}=p-p_{0}$ at a fixed instant of time (long after the excitation pulse has decayed and the initial acoustic wave generated by the pulse has passed out of the computational domain). The region between the contour lines is shaded according to the contour levels. The figure shows a clear and well defined region of high intensity within a sector of approximately $30^{\circ}$ on both sides of the the jet axis corresponding to the Mach angle for this jet. The relatively constant spacing between the outgoing waves suggests a relatively peaked spectrum, which is indeed the case as will be seen below. Note that the pressure levels are considerably higher than in the surrounding regions. The waves within the Mach cone appear to originate from a source located downstream of the jet exit. More detailed examination indicates that the waves originate from a location in the potential core of the jet. Upstream of the Mach cone, the radiation is much weaker as indicated by the contour shading and exhibits a more continuous spectrum as indicated by the lack of a regular spacing between contours. Furthermore, there are small scale structures indicating a preferred upstream radiation for high frequencies. In contrast, large scale pressure disturbances propagate in a region confined to the jet axis. This figure, although at a fixed instant of time, is typical of the behavior exhibited by $\tilde{p}$ after the excitation pulse has decayed. The intense radiation is a manifestation of Mach wave radiation generated by the expansion and contraction of disturbances near the nozzle lip forming a cellular structure in the jet. As these cells expand and contract through one cycle they generate Mach wave radiation propagating into the far field at the Mach angle of the flow with respect to the jet axis. The relatively peaked spectrum Mach wave radiation is superimposed on a low level, nearly continuous spectrum radiation pattern.

Figure 3 shows contours of vorticity and $\tilde{p}$ (upper two figures respectively) and the direction field for the unsteady velocity in a small region near the nozzle exit. The vorticity figure shows a train of vortices propagating along the jet axis and also along the jet boundary. Detailed examination shows that these vortices are generated at the nozzle lip. The vortices have a stretched appearance. Detailed comparison with lower Mach number jets shows that the stretching increases significantly with Mach number. We have performed grid refinements which show that the results presented here are insensitive to further grid refinements. The pressure contours (middle figure in Figure 3) shows a sequence of pressure disturbances generated just downstream of the nozzle exit. These disturbances, together with velocity disturbances (bottom figure) give rise to a cellular structure within the jet. These cells compress and expand in a nearly periodic fashion generating Mach wave radiation propagating into the far field at the Mach angle with respect to the jet axis. The Mach wave radiation is indicated by the alternating light and dark structures propagating away 
from the jet axis. The cellular structure of the pressure correlates with the cellular structure in the velocity field shown in the bottom-most figure. The cell size is approximately $3 D$ consistent with a peak Strouhal number of approximately 0.2 (see below). The Mach wave radiation is generated via cycles of contraction and expansion of the cells as they propagate downstream. The incipient formation of the characteristic diamond shaped cell structure immediately downstream of the nozzle exit is also noted.

Figure 4 shows the near field $\tilde{p}$ in both time and frequency domains. The data is taken at several locations along the jet axis. Generally, $\tilde{p}$ is largest within the potential flow core. The spectral data is plotted against Strouhal number $S t=f D / U_{j}$ where $f$ is the frequency and $U_{j}$ is the jet exit velocity. The spectrum is plotted in decibels and normalized so that the largest value on all four of the graphs corresponds to a decibel level of zero. Near the nozzle exit, $\tilde{p}$ has a relatively peaked spectrum centered around a peak Strouhal number, $S t_{*} \simeq 0.2$, together with bands corresponding to harmonics. This frequency will be referred to as the jet frequency and is close to the peak frequency observed in experiments for jets in this Mach number range.[9] The levels of the harmonics relative to the fundamental decrease with increasing downstream distance. The peak pressure occurs in the potential core of the jet. As downstream distance increases, the spectrum broadens toward the low end and the level of the low frequencies below the jet frequency increases. The spectrum for $x / D=15$ represents the spectrum of the large scale structures in the jet which propagate and eventually decay beyond the potential core (refer to Figure 2). Note that from Figure 2 very little sound is generated beyond $10 \mathrm{D}$.

The far field $\tilde{p}$ is shown in Figure 5, with data taken on a circle of radius $30 D$ centered on the source location (very near the nozzle exit). The data shows peak radiation for angles near $30^{\circ}$ consistent with Figure 2 (observe that the time trace for $30^{\circ}$ is plotted using a different scale than for the other angles). The spectra are again normalized so that the maximum of all four spectra in the figure corresponds to $0 \mathrm{~dB}$. The transition from the well defined spectral peaks at $30^{\circ}$ to a smaller, nearly continuous spectrum as the angle increases (i.e., tending toward the upstream direction) is apparent. Experiments with low Reynolds number jets indicate a very peaked spectrum for the Mach wave radiation. [9, 23] Higher Reynolds number jets exhibit a somewhat more continuous spectrum, presumably due to the effect of small scale turbulent fluctuations which are not accounted for in the present model. The increase in the relative high frequency content of the spectrum with increasing upstream angle is also noted.

Finally in Figure 6, the overall sound pressure level as a function of far field angle is examined. The figure shows a strong peak near $30^{\circ}$, consistent with many experimental measurements, together with a smaller peak upstream (angles near $150^{\circ}$ ) similar to the peak sometimes observed for jets in this Mach number range.[9] This peak is de-emphasized by the presence of the wall (see below).

\subsection{Installed Jet}

The behavior of the installed jet is considered next. In Figure 7, contours of $\tilde{p}$ are shown

for both the jet and radiation domain at a fixed instant of time. Note that the placement of 
the panels relative to the nozzle exit is as indicated in the figure. The Mach wave radiation below the panels, similar to that for the free jet, can be seen in the bottom figure. The Mach wave radiation is less regular than for the free jet, indicating a significant effect of the wall on the directivity even in the region below the panels. There are large pressure disturbances near the wall in the jet domain. Detailed examination shows that these disturbances convect downstream.

Many of the directivity features in the jet domain are transmitted to the radiation domain. The most pronounced feature is a beaming from the panels at approximately $30^{\circ}$ into the radiation domain. At larger angles (i.e., pointing more toward the upstream) the radiated pressure is dominated by smaller scales (higher frequencies), also similar to the jet domain. Note also a virtual zone of silence in the radiation domain for angles near $180^{\circ}$.

Examination of the near field and far field $\tilde{p}$ in the jet domain indicates a behavior similar to the free jet. Therefore this data is not shown here. However, Figure 8 shows the jet domain far field directivity taken at points below the jet axis (i.e., away from the wall). The results show a significant de-emphasis of the peak at $150^{\circ}$ induced by the presence of the wall.

The panel response and radiation are considered next. In computing the response of the panels, significant effort was made to insure that the response was due to the long time behavior of the jet rather than to the initial wave generated by the excitation pulse. This is more critical than for the free jet due to the low damping of the panels. The panels were kept rigid (i.e. the loading pressure - difference in pressure between the radiation and jet domains - was set to zero) up to a certain time. This time was chosen such that the initial wave generated by the excitation pulse had passed away from the panels and could no longer serve to force the panels. The panels were then allowed to vibrate as the loading pressure was slowly increased to the true pressure difference. Thus the panel response does not include effects from the excitation pulse or from an abrupt switching on of the loading pressure.

The long time pressure incident on the six panels $\left(\tilde{p}_{I}\right)$ in both the time and frequency domain are examined in Figure 9 and Figure 10. The data is taken at the center of each panel and the spectra are normalized to zero decibels by the maximum for all of the panels. A significant change is seen in the character of $\tilde{p}_{I}$ as the panel location shifts downstream. For the most upstream panels, (panels 1 and 2) $\tilde{p}_{I}$ is at a lower level and is essentially continuous. As the downstream location increases, the spectra become increasingly peaked. The peak frequency corresponds to the jet frequency Strouhal number, $S t_{*}$. Note, for example, that for panels $4-6$, the spectrum is dominated by $S t_{*}$ and its harmonic. Furthermore, the level of the forcing is nearly two orders of magnitude greater for the downstream panels than for the upstream panels. The results show that the Mach wave radiation results in an extreme sensitivity of the panel loading to the location of the panels for $\tilde{p}_{I}$.

The result of this sensitivity on the panel response is investigated next. In Figure 11 and Figure 12 the vertical velocity $v$ at the panel centers is plotted in both the time and frequency domain. The panel response increases by almost two orders of magnitude from panel 1 to panel 6, consistent with the increased loading due to the Mach wave radiation in the jet domain. Furthermore, for the panels outside of the Mach cone, the spectrum shows no single preferred frequency. The spectrum is essentially continuous and low in amplitude. For the panels located within the cone, however, there is a strong peak near the jet frequency consistent with the loading. The upper portion of the spectrum is seen to increase relative 
to that of $\tilde{p}_{I}$. Furthermore, the upper portion of the spectrum exhibits more of a banded nature, consistent with the behavior for lower speed jets.[1, 3]

Next, the pressure in the radiation domain is considered. The transmitted pressure (taken at the center of the panels) exhibits a behavior similar to that shown for $v$ and is not shown. Shown instead is the radiation domain pressure taken along the line $y=25 D$ in the radiation domain (indicated by the small arrow in Figure 7). Figure 13 shows the radiated pressure at four different locations along this line. There is a large increase in level for points within the radiation beam (see the upper contour plot in Figure 7). Note that the data is taken along a line, not a circle, and thus the increase in level with $x$ occurs in spite of the radial decay which should reduce the radiated pressure for large values of $x$. Also note the emergence of a distinct peak near the jet frequency for points within the beam (e.g., the third graph in the figure). This is also apparent in the fourth graph but is masked somewhat in view of the fact that less data is available over the given time interval due to the large value of $x$.

Finally in Figure 14, the overall sound pressure level is plotted as a function of $x$ along this line. The figure shows the analog of the jet domain beaming in the radiation domain, consistent with the visualization in Figure 7.

\section{Conclusion}

The full unsteady fluid dynamic field, including the far field acoustic pressure, in an excited supersonic jet has been computed for the case where the jet is free and for the case where the jet is installed near an array of flexible aircraft type panels. Only the long time response of the jet is considered. This response is dominated by a very pronounced and intense Mach wave radiation generated by a succession of cellular structures formed by instability waves in the jet column. The predominant features of the long time pressure field in the jet are:

1. Pressure and velocity disturbances initiated near the nozzle lip give rise to a cellular structure within the jet. These cells expand and contract as they propagate downstream, generating acoustic waves which propagate into the far field at the Mach angle of the jet.

2. This Mach wave radiation is the most pronounced feature of the jet far field and is characterized by a relatively peaked spectrum with a peak Strouhal number of approximately 0.2 (together with harmonics), close to observations for jets in this Mach number range. The peak frequencies arise even though the jet is subject to transient (broadband) forcing. Thus there is a particular frequency, the jet frequency, associated with the long time response of the jet.

3. Sound radiation for angles greater than the Mach angle is at a much lower level, with a relative increase in high frequencies with increasing upstream direction.

4. Near field pressure along the jet axis is characterized by a relatively peaked spectrum near the potential core but becomes increasingly continuous with increasing downstream distance. 
5. Vortices associated with the pressure instability waves are significantly stretched as compared with lower Mach number jets.

The following conclusions have been shown for the installed jet:

1. The intense radiation of sound in the jet domain in the direction of the Mach angle observed in the free jet persists for the installed jet. However, the presence of the wall causes some distortion.

2. The loading of the panels depends crucially on location. Panels within the Mach cone are subject to a high level loading which peaks near the jet frequency Strouhal number, $S t_{*}$. Panels upstream of the Mach cone are subject to a low level loading with a nearly continuous spectrum.

3. The panels within the Mach cone exhibit a much larger response than panels outside of the cone, consistent with the incident pressure. The panel response is also peaked at the jet frequency.

4. The radiated pressure exhibits a beaming at roughly the same angle from the wall as the beaming in the jet domain. The pressure for points in this radiation beam exhibits a spectral peak close to the jet frequency.

\section{References}

[1] McGreevy, J.L., Bayliss, A. and Maestrello, L., "Interaction of Jet Noise with a Nearby Panel Assembly," AIAA Journal, Vol. 33, 1995, pp. 577-585.

[2] Bayliss, A., Maestrello, L., McGreevy, J.L. and Fenno, C.C., "Forward Motion Effects on Jet Noise, Panel Vibration, and Radiation," AIAA Journal, Vol. 34, 1996, pp. 11031110.

[3] Fenno, C.C., Bayliss, A. and Maestrello, L., "Panel-Structure Response to Acoustic Forcing by a Nearly Sonic Jet," AIAA Paper 96-1762, 1996 (to appear in AIAA Journal).

[4] Lighthill, M.J., "On Sound Generated Aerodynamically-I, General Theory," Proceedings of the Royal Society, Vol. A222, 1954, pp. 1-32.

[5] Lilley, G.M., "Theory of Turbulence Generated Jet Noise: Generation of Sound in a Mixing Region," U.S. Air Force Technical Report AFAPL-TR-72-53, IV, Wright Patterson AFB, 1972.

[6] Ribner, H.S., "Dryden Lecture, Perspectives on Jet Noise," AIAA Journal, Vol. 19, 1981, pp. 1513-1526.

[7] Ribner, H.S., "An Extension of the Lighthill Theory of Jet Noise to Encompass Refraction and Shielding," NASA Technical Memorandum 110163, 1995.

[8] Ffowcs Williams, J.E. and Kempton, A. J., "The Noise from the Large-Scale Structure of a Jet," J. Fluid Mech., Vol. 84, 1975, pp. 673-694. 
[9] Seiner, J.M., "Fluid Dynamics and Noise Emission Associated with Supersonic Jets", in Studies in Turbulence, Springer Verlag, eds. T. B. Gatski, S. Sarkar and C. G. Speziale, pp. 297-323.

[10] Crow, S. and Champagne, F., "Orderly Structure in Jet Turbulence," Journal of Fluid Mechanics, Vol. 48, 1971, pp. 457-591.

[11] Kibens, V., "Discrete Noise Spectrum Generated by an Acoustically Excited Jet," AIAA Journal, Vol. 18, 1980, pp. 434-441.

[12] Maestrello, L., Bayliss, A. and Turkel, E., "On the Interaction of a Sound Pulse with the Shear Layer of an Axisymmetric Jet," Journal of Sound and Vibration, Vol. 74, 1981, pp. 281-301.

[13] Bechert, D.W. and Pfizenmaier, E., "On the Amplification of Broadband Jet Noise by Pure Tone Excitation," Journal of Sound and Vibration, Vol. 43, 1975, pp. 581-587.

[14] Huerre, P. and Monkewitz, P.A., "Local and Global Instabilities in Spatially-Developing Flows," Annual Review of Fluid Mechanics, Vol. 22, 1990, pp. 473-537.

[15] Michalke, A. and Hermann, G., "On the Inviscid Instability of a Circular Jet With External Flow," Journal of Fluid Mechanics, Vol. 114, 1982, pp. 343-359.

[16] Michalke, A., "Survey on Jet Instability Theory," Progress in Aerospace Science, Vol. 21, 1984, pp. 159-199.

[17] Bayliss, A., Maestrello, L. and Turkel, E., "On the Interaction of a Sound Pulse With the Shear Layer of an Axisymmetric Jet, III: Non-Linear Effects," Journal of Sound and Vibration, Vol. 107, 1986, pp. 167-175.

[18] Maestrello, L. and Bayliss, A., "Flowfield and Far Field Acoustic Amplification Properties of Heated and Unheated Jets," AIAA Journal, Vol. 20, 1982, pp. 1539-1546.

[19] Frendi, A., Maestrello, L. and Bayliss, A., "On the Coupling Between a Supersonic Boundary Layer and a Flexible Surface," AIAA Journal, Vol. 31, 1993, pp. 708-713.

[20] Frendi, A., Maestrello, L. and Bayliss, A., "Coupling Between Plate Vibration and Acoustic Radiation," Journal of Sound and Vibration, Vol. 177, No. 2, 1994, pp. 207226.

[21] Mankbadi, R., Hayder, M. and Povinelli, L., "The Structure of Supersonic Jet Flow and Its Radiated Sound," AIAA Journal, Vol. 32, 1994, pp. 897-906.

[22] Gottlieb, D. and Turkel, E., "Dissipative Two-Four Methods For Time-Dependent Problems," Mathematics of Computation, Vol. 30, 1976, pp. 703-723.

[23] McLaughlin, D.K., Morrison, G.L. and Trout, T.R., "Reynolds Number Dependence in Supersonic Jet Noise," AIAA Journal, Vol. 15, 1977, pp. 526-532. 


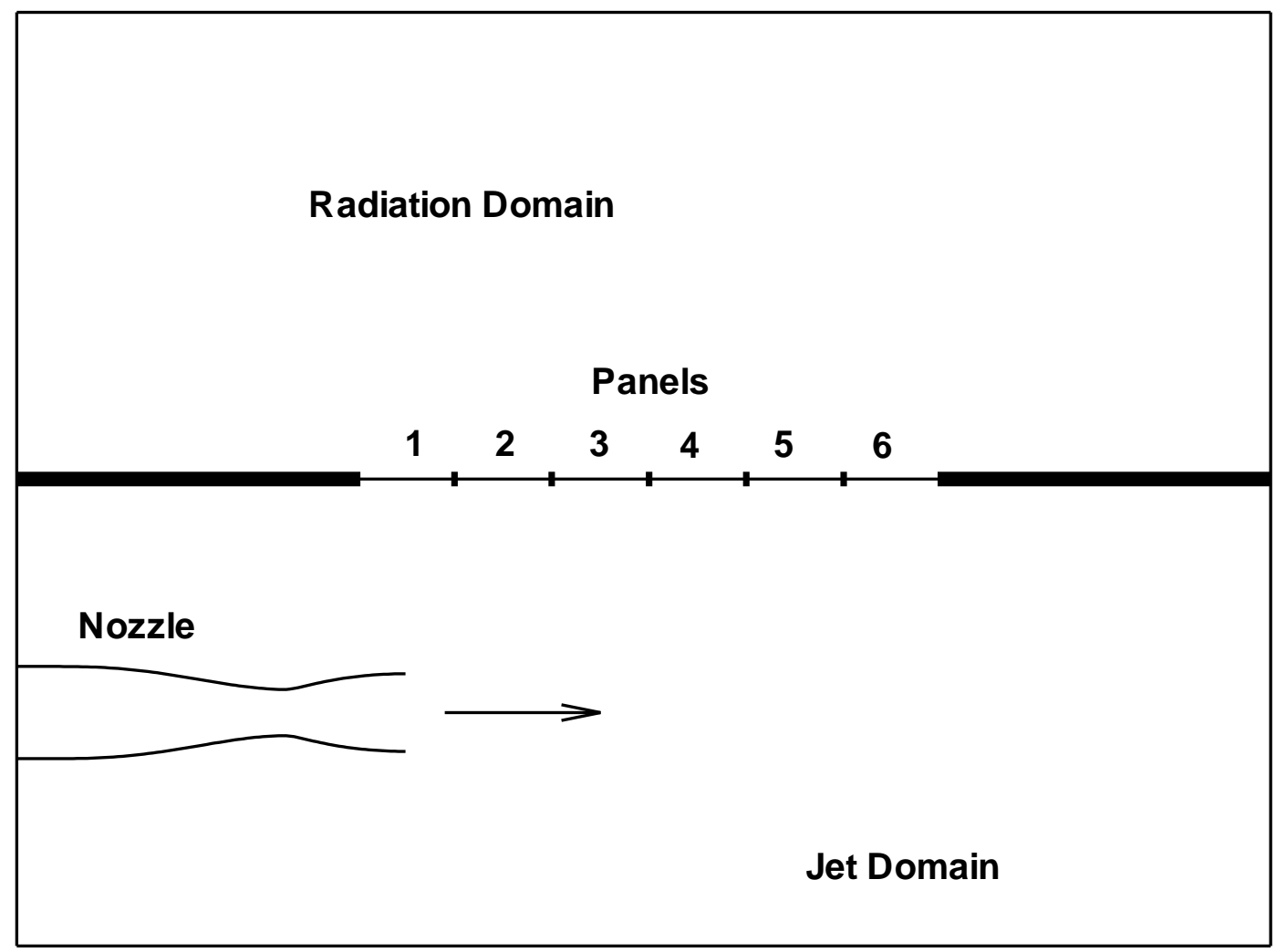

Figure 1: Computational domain for installed jet. 


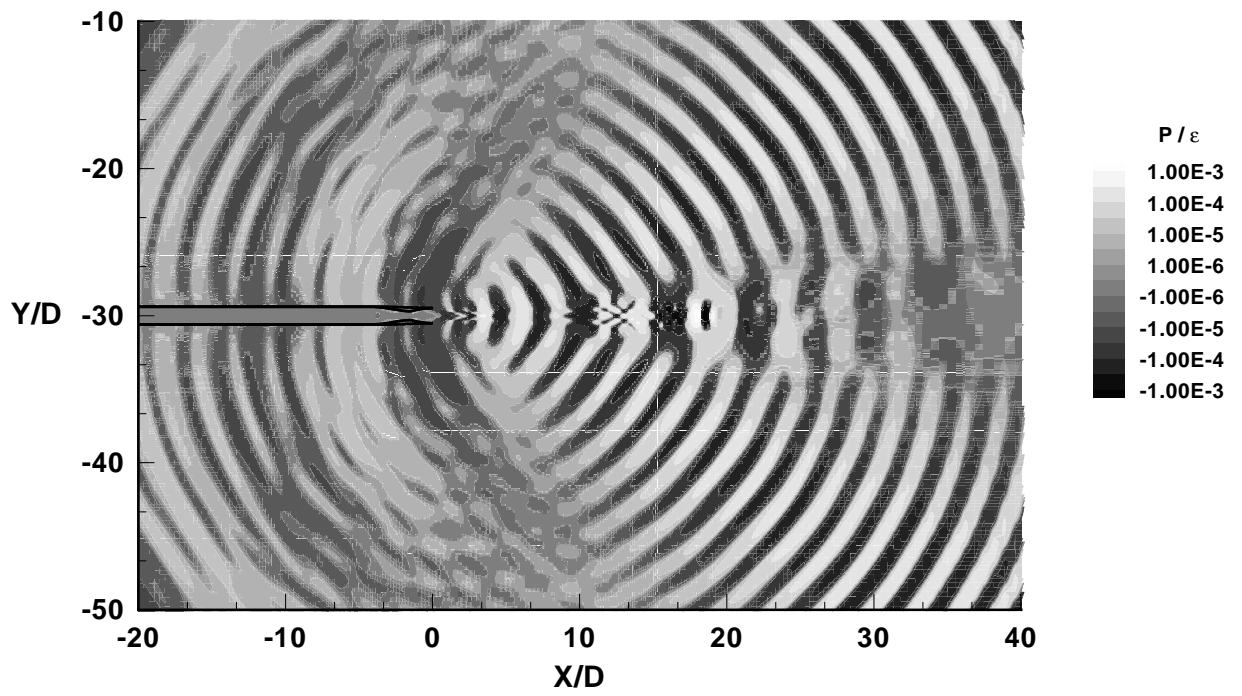

Figure 2: Contours for $\tilde{p}$ for free jet. 

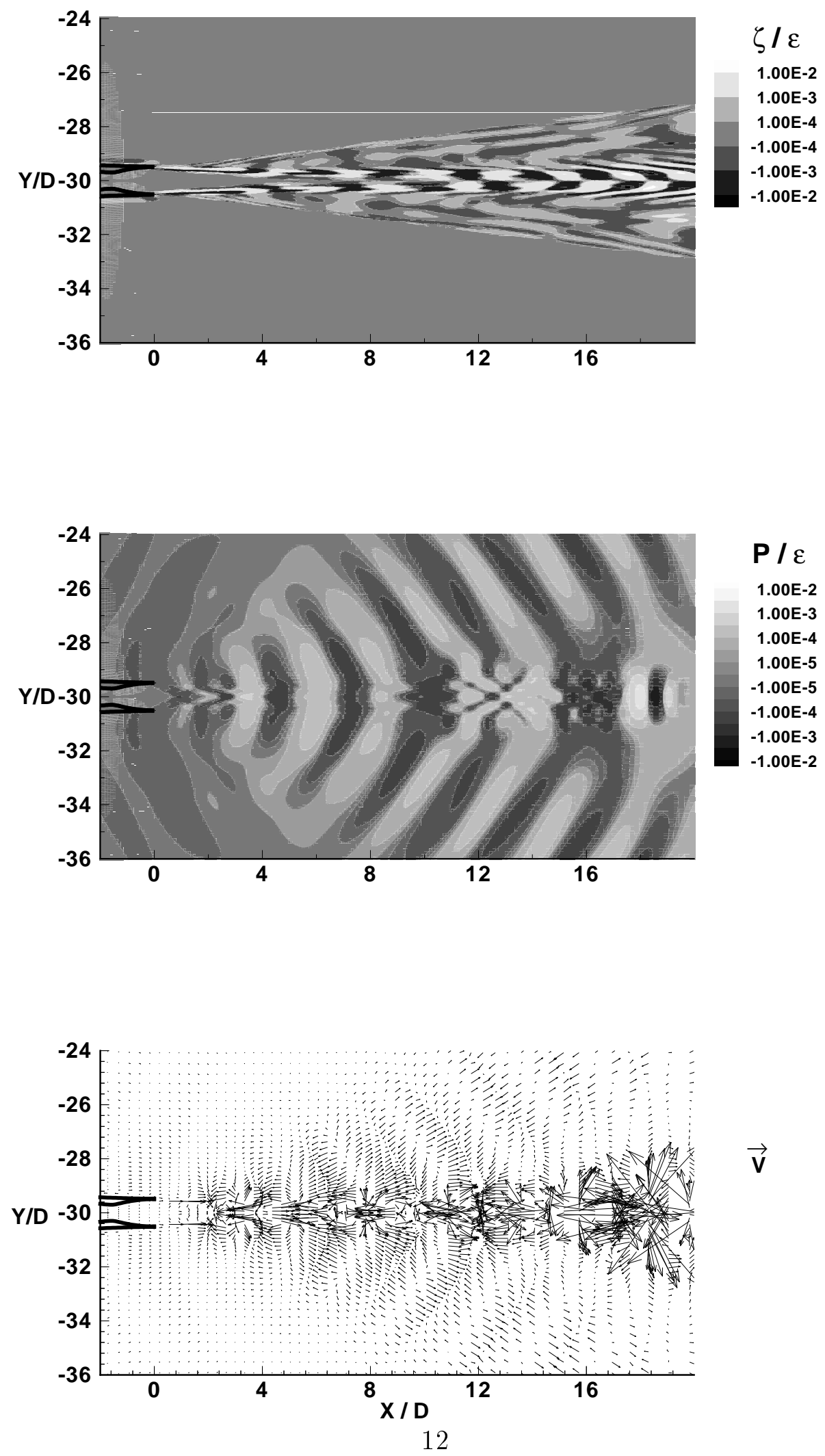

Figure 3: Contours for vorticity (upper figure) $\tilde{p}$ (middle figure) and unsteady velocity field (lower figure) for region near nozzle exit for free jet. 

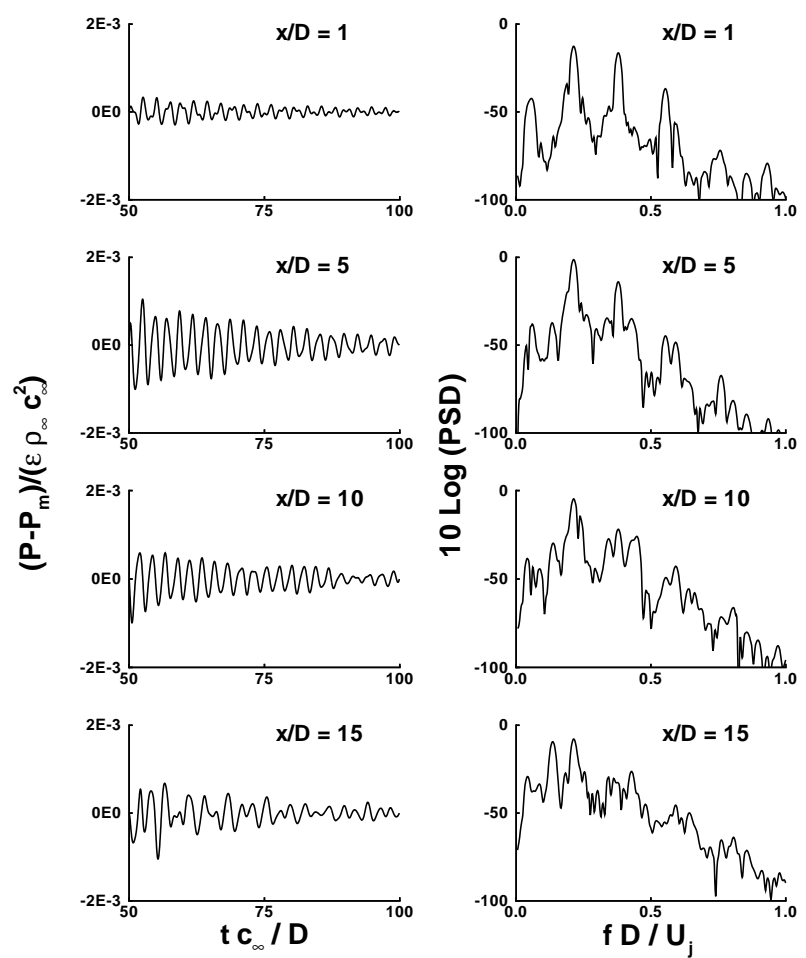

Figure 4: Near field $\tilde{p}$ in both time and frequency domains for free jet. 

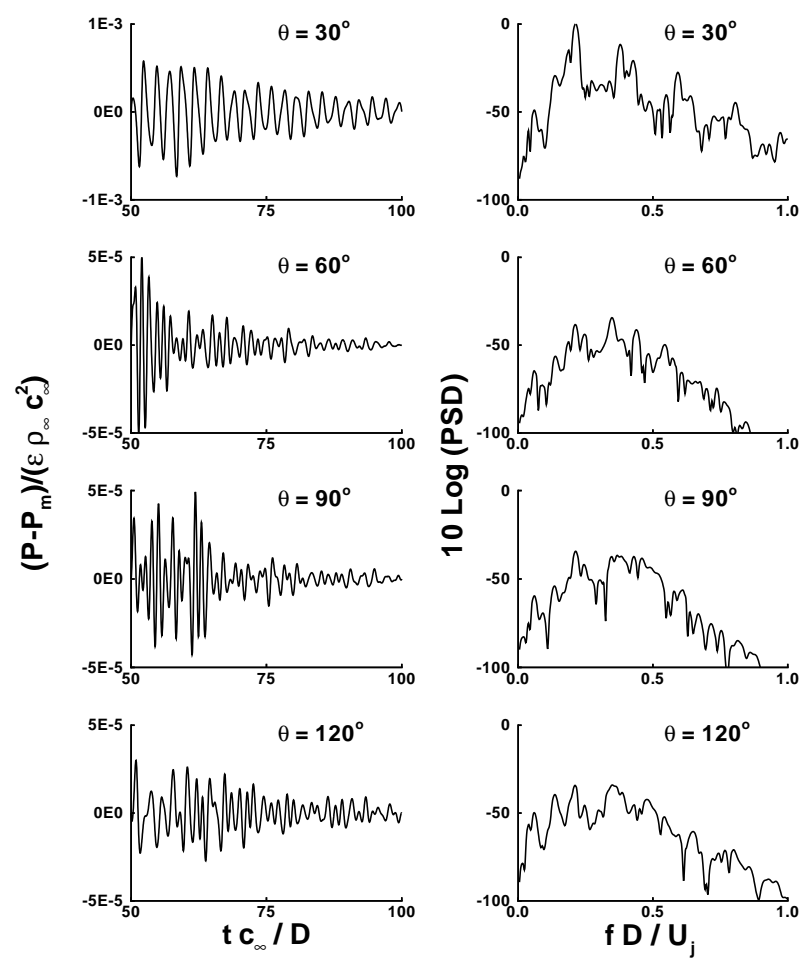

Figure 5: Far field $\tilde{p}$ in both time and frequency domains for free jet. 


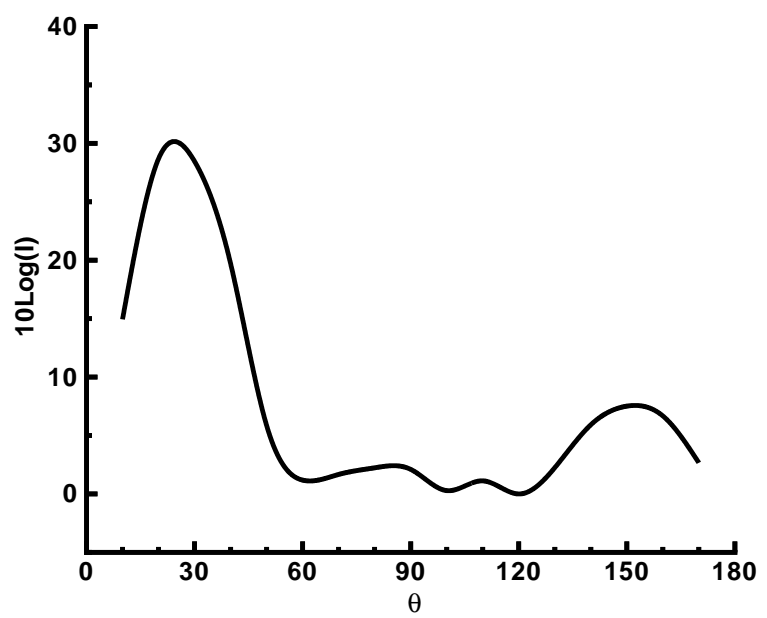

Figure 6: Far field directivity for free jet 


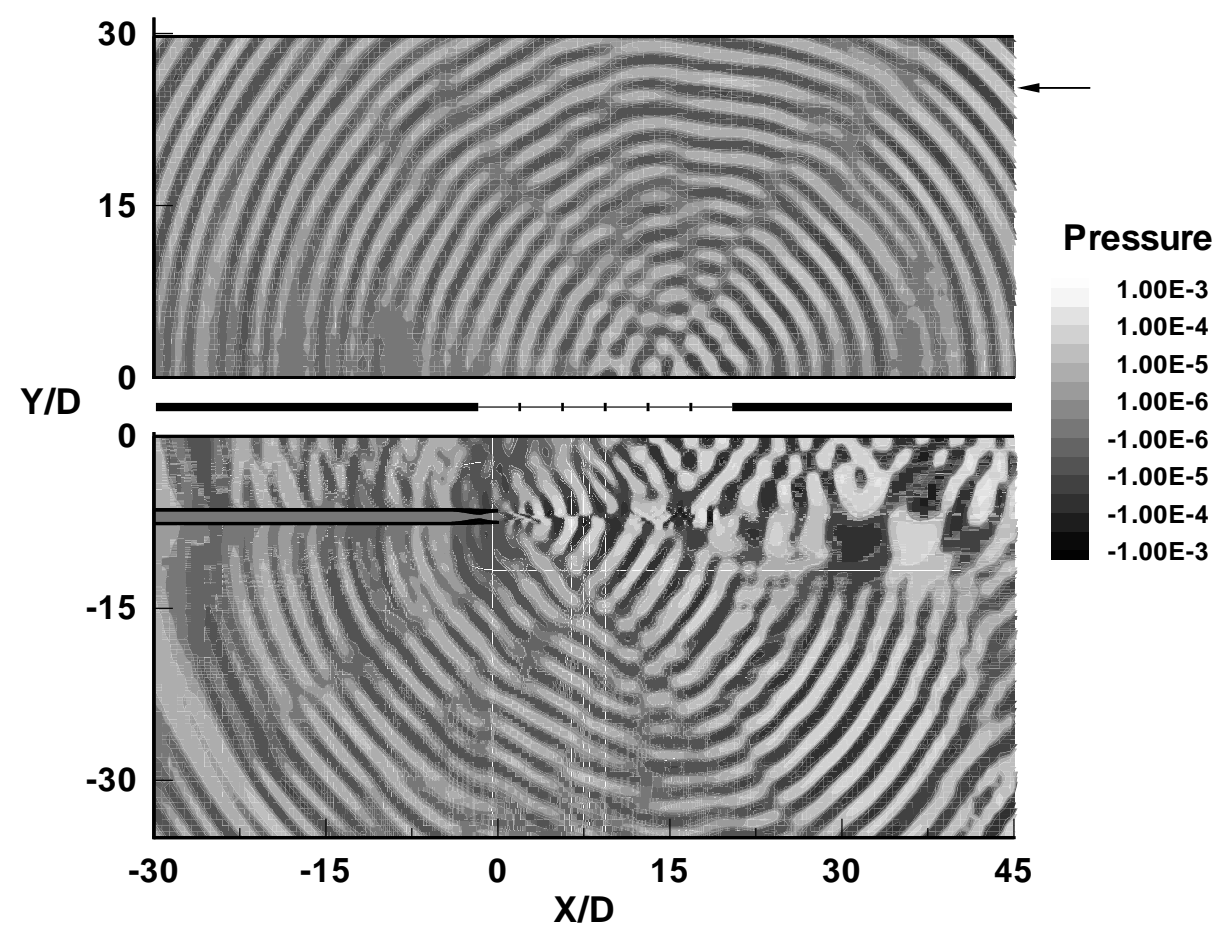

Figure 7: Contours for $\tilde{p}$ for installed jet. Both jet domain (lower figure) and radiation domain (upper figure) are shown. 


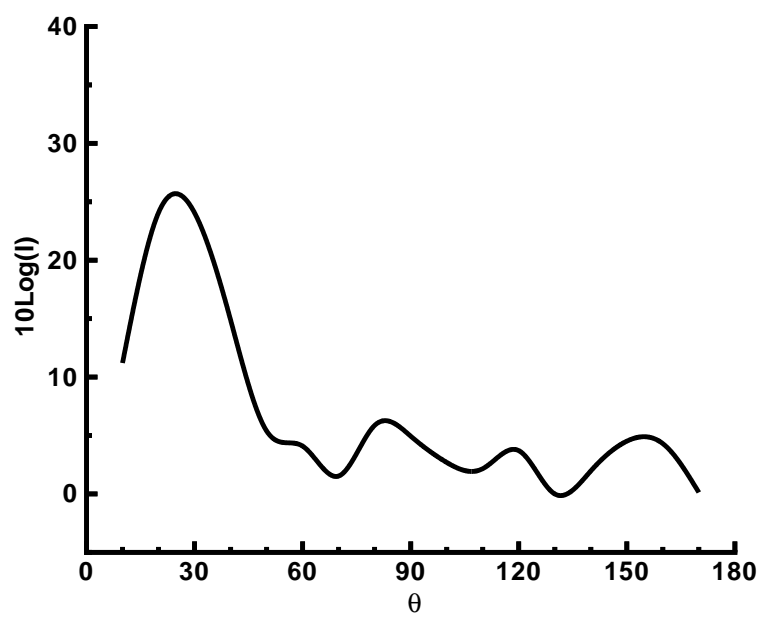

Figure 8: Far field directivity for installed jet. 

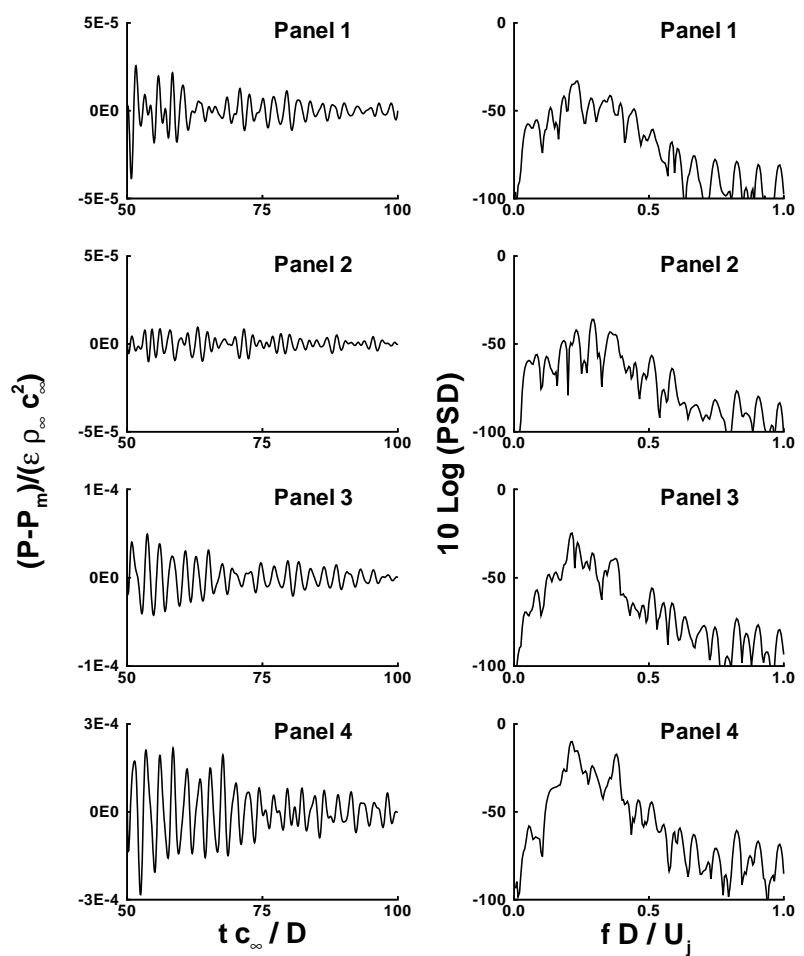

Figure 9: Incident pressure (from jet domain) at the panel centers (panels 1-4). 

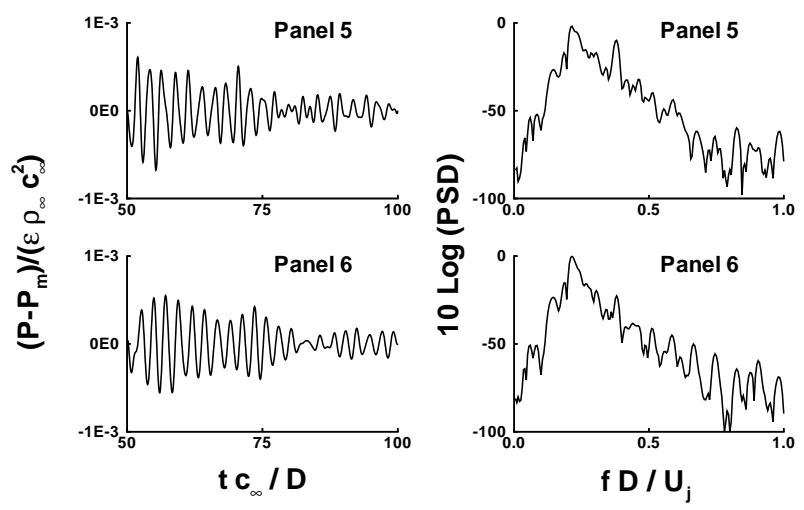

Figure 10: Incident pressure (from jet domain) at the panel centers (panels 5 and 6). 

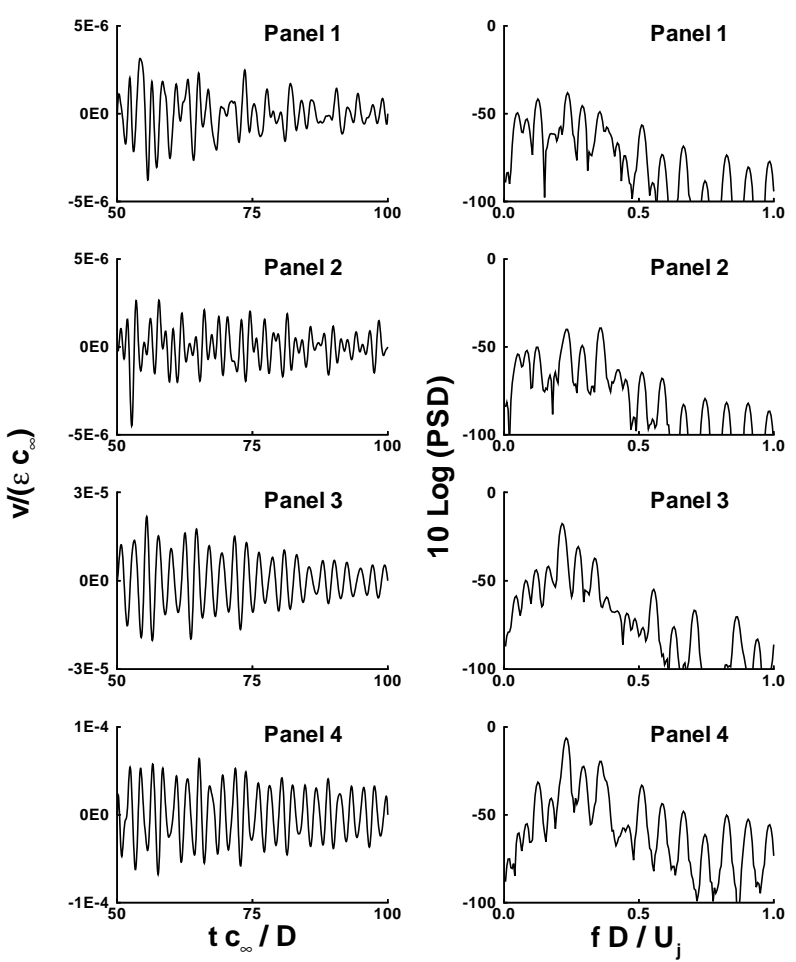

Figure 11: $v$ at the panel centers (panels 1-4). 

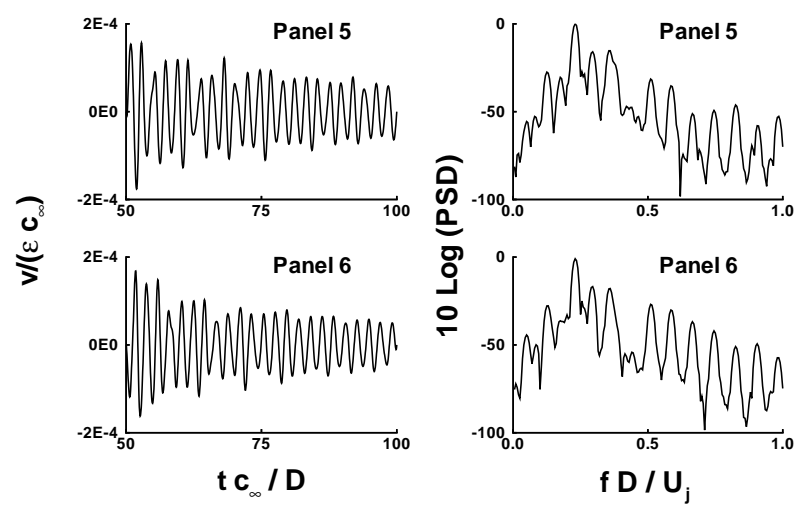

Figure 12: $v$ at the panel centers (panels 5 and 6 ). 

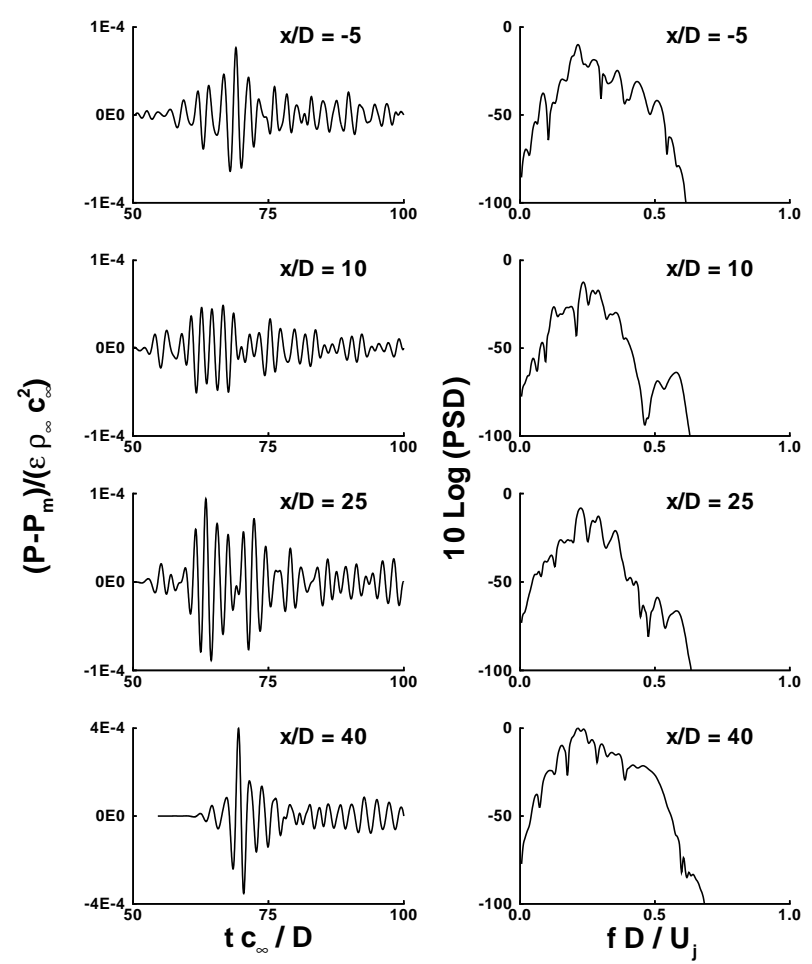

Figure 13: $\tilde{p}$ at four points along a line in the radiation domain. 


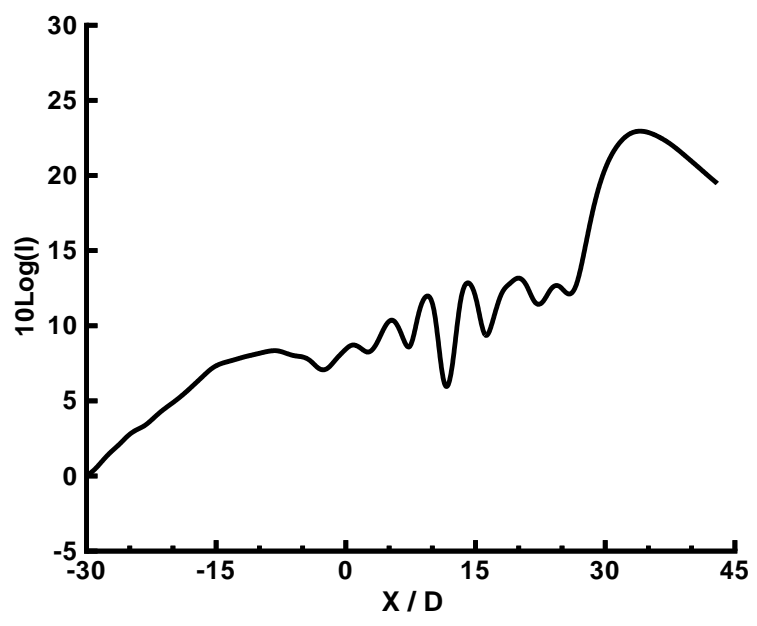

Figure 14: Overall sound pressure level as a function of $x$ along a line in the radiation domain. 